\title{
A CHARACTERIZATION OF CHAOTIC ORDER
}

\author{
CHANGSEN YANG AND FUGEN GAO
}

Received 15 November 2005; Accepted 4 January 2006

The chaotic order $A \gg B$ among positive invertible operators $A, B>0$ on a Hilbert space is introduced by $\log A \geq \log B$. Using Uchiyama's method and Furuta's Kantorovich-type inequality, we will point out that $A \gg B$ if and only if $\left\|B^{p} A^{-p / 2} B^{-p / 2}\right\| A^{p} \geq B^{p}$ holds for any $0<p<p_{0}$, where $p_{0}$ is any fixed positive number. On the other hand, for any fixed $p_{0}>0$, we also show that there exist positive invertible operators $A, B$ such that $\left\|B^{p} A^{-p / 2} B^{-p / 2}\right\| A^{p} \geq B^{p}$ holds for any $p \geq p_{0}$, but $A \gg B$ is not valid.

Copyright (C) 2006 C. Yang and F. Gao. This is an open access article distributed under the Creative Commons Attribution License, which permits unrestricted use, distribution, and reproduction in any medium, provided the original work is properly cited.

\section{Introduction}

In what follows, a capital letter means a bounded linear operator on a complex Hilbert space $H$. An operator $T$ is said to be positive, in symbol $T \geq 0$ if $(T x, x) \geq 0$ for all $x \in H$. In particular, we denote by $A>0$ if $A \geq 0$ is invertible. By the operator monotonicity of the logarithmic function, we know that $A \geq B>0$ implies the chaotic order $A \gg B$. For the chaotic order, several characterizations were shown by many authors, for example, $[1-3,6]$. The following well-known results about chaotic order were obtained.

Theorem $1.1[1,2]$. Let $A$ and $B$ be positive invertible operators. Then the following properties are mutually equivalent:

(i) $\log A \geq \log B$;

(ii) $\left(B^{p / 2} A^{p} B^{p / 2}\right)^{1 / 2} \geq B^{p}$ for all $p \geq 0$;

(iii) $\left(B^{r / 2} A^{p} B^{r / 2}\right)^{r /(p+r)} \geq B^{r}$ for all $p \geq 0$ and $r \geq 0$.

Theorem 1.2 Kantorovich type inequalities [3]. Let $A>0$ and for positive numbers $M, m$, $M \geq B \geq m>0$. Then the following parallel statements hold. Moreover, (ii) can be derived from (i). 
2 A characterization of chaotic order

(i) $A \geq B$ implies $\left(\left(M^{p-1}+m^{p-1}\right)^{2} /\left(4 m^{p-1} M^{p-1}\right)\right) A^{p} \geq B^{p}$ for all $p \geq 2$.

(ii) $\log A \geq \log B$ implies $\left(\left(M^{p}+m^{p}\right)^{2} /\left(4 m^{p} M^{p}\right)\right) A^{p} \geq B^{p}$ for all $p \geq 0$.

Theorem 1.3 [6]. Let $A$ and $B$ be positive invertible operators. Then $A \geq B>0$ if and only if $\left\|B^{p-1} A^{-(p-2) / 2} B^{-p / 2}\right\| A^{p-1} \geq B^{p-1}$ for all $p \geq 2$.

As a parallel statement of Theorem 1.3, we point out the following result on the chaotic order of two positive invertible operators.

Theorem 1.4. Let $A$ and $B$ be positive invertible operators. Then for a fixed $p_{0}>0$, the following assertions are mutually equivalent:

(i) $A \gg B$;

(ii) $\left\|B^{p} A^{-p / 2} B^{-p / 2}\right\| A^{p} \geq B^{p}$ holds for all $p>0$;

(iii) $\left\|B^{p} A^{-p / 2} B^{-p / 2}\right\| A^{p} \geq B^{p}$ holds for any $p \in\left(0, p_{0}\right)$.

On the other hand, we will prove that the condition $p \in\left(0, p_{0}\right)$ in Theorem 1.4 is essential as follows.

Theorem 1.5. For a fixed $p_{0}>0$, there exist positive invertible operators $A, B$ such that $\left\|B^{p} A^{-p / 2} B^{-p / 2}\right\| A^{p} \geq B^{p}$ holds for any $p \geq p_{0}$, but $A \gg B$ is not valid.

\section{The proofs of the main results}

To give a proof of Theorem 1.4, we also need the following well-known theorem used in [3] which is essentially the same as [5].

Theorem $2.1[3,5]$. Let $X>0$, then $\lim _{n \rightarrow \infty}(I+\log X / n)^{n}=X$.

Proof of Theorem 1.4. (i) $\Rightarrow$ (ii) Suppose that $\log A \geq \log B$. Let $p>0$, then for sufficiently large $n$, we have $I+\log A / n \geq I+\log B / n>0$ and $n p \geq 2$. Put $A_{1}=I+\log A / n$ and $B_{1}=$ $I+\log B / n$. Then we have $A_{1} \geq B_{1}>0$ and applying Theorem 1.3 , the following inequality holds:

$$
\left\|B_{1}^{n(p-1 / n)} A_{1}^{n(-(p-2 / n) / 2)} B_{1}^{n(-p / 2)}\right\| A_{1}^{n(p-1 / n)} \geq B_{1}^{n(p-1 / n)}
$$

for all $n p \geq 2$. By Theorem 2.1, we have $A_{1}^{n} \rightarrow A$ and $B_{1}^{n} \rightarrow B$ as $n \rightarrow \infty$. Hence let $n \rightarrow \infty$ in (2.1), then we obtain $\left\|B^{p} A^{-p / 2} B^{-p / 2}\right\| A^{p} \geq B^{p}$ holds for all $p>0$;

(ii) $\Rightarrow$ (iii) Obvious.

(iii) $\Rightarrow$ (i) Let $0<p<p_{0}$ and $\lambda_{p}=\left\|B^{p} A^{-p / 2} B^{-p / 2}\right\|$. Then $B^{p} \leq \lambda_{p} A^{p}$ by (iii). By L-H theorem, we also have $B^{p / 2} \leq \lambda_{p}^{1 / 2} A^{p / 2}$, thus $B^{3 p / 2} \leq \lambda_{p}^{1 / 2} B^{p / 2} A^{p / 2} B^{p / 2}$. Now suppose that $0<$ $m \leq B \leq M$. So $0<m^{3 p / 2} \leq B^{3 p / 2} \leq M^{3 p / 2}$. Applying (i) of Theorem 1.2, we obtain

$$
B^{3 p} \leq \frac{\left(M^{3 p / 2}+m^{3 p / 2}\right)^{2}}{4 M^{3 p / 2} m^{3 p / 2}} \lambda_{p}\left(B^{p / 2} A^{p / 2} B^{p / 2}\right)^{2} .
$$


Hence

$$
B^{2 p} \leq \frac{\left(M^{3 p / 2}+m^{3 p / 2}\right)^{2}}{4 M^{3 p / 2} m^{3 p / 2}} \lambda_{p} A^{p / 2} B^{p} A^{p / 2} .
$$

By (2.3) and $\lambda_{p}=\left\|B^{-p / 2} A^{-p / 2} B^{2 p} A^{-p / 2} B^{-p / 2}\right\|^{1 / 2}$, we have

$$
\lambda_{p}^{2} \leq \frac{\left(M^{3 p / 2}+m^{3 p / 2}\right)^{2}}{4 M^{3 p / 2} m^{3 p / 2}} \lambda_{p}
$$

So

$$
\lambda_{p} \leq \frac{\left(M^{3 p / 2}+m^{3 p / 2}\right)^{2}}{4 M^{3 p / 2} m^{3 p / 2}} .
$$

Therefore

$$
B^{p} \leq \frac{\left(M^{3 p / 2}+m^{3 p / 2}\right)^{2}}{4 M^{3 p / 2} m^{3 p / 2}} A^{p} .
$$

By (2.6), we also have

$$
\log B \leq \frac{1}{p} \log \frac{\left(M^{3 p / 2}+m^{3 p / 2}\right)^{2}}{4 M^{3 p / 2} m^{3 p / 2}}+\log A .
$$

Let $p \rightarrow 0$, we obtain (i).

To prove Theorem 1.5, we first cite the following simple inequalities.

Lemma 2.2. Let $a, b, d$ be three positive numbers, then

(i) $b \leq\left\|\left(\begin{array}{ll}a & b \\ b & d\end{array}\right)\right\|$,

(ii) $\left(\begin{array}{ll}a & b \\ b & d\end{array}\right) \leq(a+b+d) I$.

Proof of Theorem 1.5. Suppose $p_{0}>0$. Let $A=\left(\begin{array}{cc}9 / 5 & -2 / 5 \\ -2 / 5 & 6 / 5\end{array}\right)$, and $B=\left(\begin{array}{ll}2 & 0 \\ 0 & \varepsilon\end{array}\right)$, where $\varepsilon \in(0,(1 /$ 2) $\left.\left[\left(2-2^{1-p_{0} / 2}\right)^{2} /\left(7+3 \cdot 2^{-p_{0}}\right)^{4}\right]^{1 / p_{0}}\right)$.

Note that $A=U^{*}\left(\begin{array}{ll}2 & 0 \\ 0 & 1\end{array}\right) U$, where $U=(1 / \sqrt{5})\left(\begin{array}{cc}-2 & 1 \\ 1 & 2\end{array}\right)$ is a unitary operator, by a simple computation, we have

$$
\left.\begin{array}{rl}
B^{-p / 2} A^{-p / 2} B^{2 p} A^{-p / 2} B^{-p / 2} & \left(2-2^{1-p / 2}\right)\left[\begin{array}{rr}
\left(1+2^{2-p / 2}\right)^{2} 2^{p}+2^{-p} \varepsilon^{2 p}\left(2-2^{1-p / 2}\right)^{2} & \varepsilon^{p / 2} \\
+\frac{\varepsilon^{3 p / 2}\left(4+2^{-p / 2}\right)}{2^{p / 2}}
\end{array}\right] \\
\left(2-2^{1-p / 2}\right)\left[\begin{array}{r}
\frac{2^{3 p / 2}\left(1+2^{2-p / 2}\right)}{\varepsilon^{p / 2}} \\
\left.+\frac{\varepsilon^{3 p / 2}\left(4+2^{-p / 2}\right)}{2^{p / 2}}\right]
\end{array}\right. & 2^{2 p} \varepsilon^{-p}\left(2-2^{1-p / 2}\right)^{2}+\varepsilon^{p}\left(4+2^{-p / 2}\right)^{2}
\end{array}\right) .
$$


4 A characterization of chaotic order

Applying (i) of Lemma 2.2, we obtain

$$
\begin{aligned}
\left\|B^{-p / 2} A^{-p / 2} B^{2 p} A^{-p / 2} B^{-p / 2}\right\|^{1 / 2} \\
\geq \frac{1}{5}\left\{\left(2-2^{1-p / 2}\right)\left[\frac{2^{3 p / 2}\left(1+2^{2-p / 2}\right)}{\varepsilon^{p / 2}}+\frac{\varepsilon^{3 p / 2}\left(4+2^{-p / 2}\right)}{2^{p / 2}}\right]\right\}^{1 / 2} \\
\geq \frac{\varepsilon^{-p / 4} 2^{3 p / 4}\left(2-2^{1-p / 2}\right)^{1 / 2}}{5} \geq \frac{\varepsilon^{-p / 4} 2^{3 p / 4}\left(2-2^{1-p_{0} / 2}\right)^{1 / 2}}{5} .
\end{aligned}
$$

On the other hand, we can compute that

$$
A^{-p / 2} B^{p} A^{-p / 2}
$$

$$
=\frac{1}{25}\left(\begin{array}{cc}
2^{p}\left(1+4 \cdot 2^{-p / 2}\right)^{2}+4 \varepsilon^{p}\left(1-2^{-p / 2}\right)^{2} & \left(1-2^{-p / 2}\right)\left[2^{p+1}\left(1+4 \cdot 2^{-p / 2}\right)\right. \\
\left(1-2^{-p / 2}\right)\left[2^{p+1}\left(1+4 \cdot 2^{-p / 2}\right)\right. & \left.+2 \varepsilon^{p}\left(4+2^{-p / 2}\right)\right] \\
\left.+2 \varepsilon^{p}\left(4+2^{-p / 2}\right)\right] & 4 \cdot 2^{p}\left(1-2^{-p / 2}\right)^{2}+\varepsilon^{p}\left(4+2^{-p / 2}\right)^{2}
\end{array}\right) \text {. }
$$

Hence by Lemma 2.2 (ii), we have

$$
\begin{aligned}
A^{-p / 2} B^{p} A^{-p / 2} \leq \frac{1}{25}\{ & 2^{p}\left(1+4 \cdot 2^{-p / 2}\right)^{2}+4 \varepsilon^{p}\left(1-2^{-p / 2}\right)^{2} \\
& +\left(1-2^{-p / 2}\right)\left[2^{p+1}\left(1+4 \cdot 2^{-p / 2}\right)+2 \varepsilon^{p}\left(4+2^{-p / 2}\right)\right] \\
& \left.+4 \cdot 2^{p}\left(1-2^{-p / 2}\right)^{2}+\varepsilon^{p}\left(4+2^{-p / 2}\right)^{2}\right\}
\end{aligned}
$$

Because $0<(2 \varepsilon)^{p_{0} / 4}<\left(2-2^{1-p_{0} / 2}\right)^{1 / 2} /\left(7+3 \cdot 2^{-p_{0}}\right)<1$, so for $p>p_{0}$,

$$
(2 \varepsilon)^{p / 4}<\frac{\left(2-2^{1-p_{0} / 2}\right)^{1 / 2}}{7+3 \cdot 2^{-p_{0}}}<1 .
$$

Therefore by (2.9), (2.11), and (2.12), we have

$$
\begin{aligned}
\left\|B^{-p / 2} A^{-p / 2} B^{2 p} A^{-p / 2} B^{-p / 2}\right\|^{1 / 2} & \geq \frac{\varepsilon^{-p / 4} 2^{3 p / 4}\left(2-2^{1-p_{0} / 2}\right)^{1 / 2}}{5} \\
& \geq \frac{2^{p}}{5}\left[7+3 \cdot 2^{-p_{0}}\right] \geq A^{-p / 2} B^{p} A^{-p / 2} .
\end{aligned}
$$


To complete the proof of Theorem 1.5 , we only prove that $\left(A B^{2} A\right)^{1 / 2} \nless A^{2}$ for very small $\varepsilon>0$ by Theorem 1.1. But by a simple computation, this is equivalent to prove

$$
\left(\begin{array}{ll}
B_{1} & B_{3} \\
B_{3} & B_{2}
\end{array}\right) \equiv\left(\begin{array}{cc}
324+4 \varepsilon^{2} & -72-12 \varepsilon^{2} \\
-72-12 \varepsilon^{2} & 16+36 \varepsilon^{2}
\end{array}\right)^{1 / 2} \nless\left(\begin{array}{cc}
17 & -6 \\
-6 & 8
\end{array}\right) .
$$

Let $A_{1}=324+4 \varepsilon^{2}, A_{2}=16+36 \varepsilon^{2}$, and $A_{3}=-72-12 \varepsilon^{2}$. By [4], if

$$
V=\frac{1}{\sqrt{A_{1}-A_{2}+2 \varepsilon_{1}}}\left(\begin{array}{cc}
\sqrt{A_{1}-A_{2}+\varepsilon_{1}} & -\sqrt{\varepsilon_{1}} \\
-\sqrt{\varepsilon_{1}} & -\sqrt{A_{1}-A_{2}+\varepsilon_{1}}
\end{array}\right),
$$

where

$$
2 \varepsilon_{1}=-A_{1}+A_{2}+\sqrt{\left(A_{1}-A_{2}\right)^{2}+4 A_{3}^{2}} .
$$

Then

$$
\left(\begin{array}{cc}
B_{1} & B_{3} \\
B_{3} & B_{2}
\end{array}\right)=V\left(\begin{array}{cc}
\sqrt{A_{1}+\varepsilon_{1}} & 0 \\
0 & \sqrt{A_{2}-\varepsilon_{1}}
\end{array}\right) V
$$

Hence

$$
B_{1}=\frac{\left(A_{1}-A_{2}+\varepsilon_{1}\right) \sqrt{A_{1}+\varepsilon_{1}}+\varepsilon_{1} \sqrt{A_{2}-\varepsilon_{1}}}{A_{1}-A_{2}+2 \varepsilon_{1}} .
$$

When $\varepsilon$ is very small, we have

$$
\begin{gathered}
2 \varepsilon_{1}=-308+32 \varepsilon^{2}+\sqrt{115600-12800 \varepsilon^{2}+o\left(\varepsilon^{2}\right)}=32+\frac{224}{17} \varepsilon^{2}+o\left(\varepsilon^{2}\right) ; \\
\varepsilon_{1}=16+\frac{112}{17} \varepsilon^{2}+o\left(\varepsilon^{2}\right) ; \quad \sqrt{A_{1}+\varepsilon_{1}}=\sqrt{340}+o(\varepsilon) ; \\
A_{1}-A_{2}+2 \varepsilon_{1}=340+o(\varepsilon) ; \quad \varepsilon_{1} \sqrt{A_{2}-\varepsilon_{1}}=o(1) .
\end{gathered}
$$

Hence by (2.18), we have $B_{1}=324 / \sqrt{340}+o(1)$. Because $324 / \sqrt{340}>17$, so (2.14) is valid for some small $\varepsilon>0$.

Therefore the proof of Theorem 1.5 is complete.

The following corollary can be derived from Theorem 1.4.

Corollary 2.3. Let $T$ be an invertible operator. Then $T$ is a log-hyponormal operator if and only if

$$
\left\|\left|T^{*}\right|^{2 p}|T|^{-p}\left|T^{*}\right|^{-p}\right\||T|^{2 p} \geq\left|T^{*}\right|^{2 p}
$$

holds for any small $p>0$. 


\section{A characterization of chaotic order}

\section{References}

[1] T. Ando, On some operator inequalities, Mathematische Annalen 279 (1987), no. 1, 157-159.

[2] M. Fujii, T. Furuta, and E. Kamei, Furuta's inequality and its application to Ando's theorem, Linear Algebra and Its Applications 179 (1993), 161-169.

[3] T. Furuta, Results under $\log A \geq \log B$ can be derived from ones under $A \geq B \geq 0$ by Uchiyama's method-associated with Furuta and Kantorovich type operator inequalities, Mathematical Inequalities \& Applications 3 (2000), no. 3, 423-436.

[4] K. Tanahashi, Best possibility of the Furuta inequality, Proceedings of the American Mathematical Society 124 (1996), no. 1, 141-146.

[5] M. Uchiyama, Some exponential operator inequalities, Mathematical Inequalities \& Applications 2 (1999), no. 3, 469-471.

[6] T. Yamazaki, Characterizations of $\log A \geq \log B$ and normaloid operators via Heinz inequality, Integral Equations and Operator Theory 43 (2002), no. 2, 237-247.

Changsen Yang: Department of Mathematics, Henan Normal University, Xinxiang, Henan 453007, China

E-mail address: yangchangsen0991@sina.com

Fugen Gao: Department of Mathematics, Henan Normal University, Xinxiang, Henan 453007, China E-mail address: gaofugen@tom.com 